\title{
Gut microbiome as a biomarker of cardiometabolic disorders
}

\author{
Anna Gózd-Barszczewska',A-D, Maria Kozioł-Montewka, ${ }^{2, E-F}$, Piotr Barszczewski, ${ }^{3, C, D}$, \\ Agata Młodzińska ${ }^{4, B-C}$, Kinga Humińska ${ }^{5, B}$ \\ ${ }^{1}$ Division of Cardiology with Cardiac Intensive Care Unit, Cardinal Stefan Wyszyński Hospital, Lublin, Poland \\ 2 Pope John Paul II State School of Higher Education, Biała Podlaska, Poland \\ ${ }^{3}$ Chair and Department of Medical Microbiology, Medical University, Lublin, Poland; Division of Gastroenterology, \\ Cardinal Stefan Wyszyński Hospital, Lublin, Poland \\ ${ }^{4}$ Genomic Laboratory, DNA Research Centre, Poznań, Poland \\ ${ }^{5}$ Genomic Laboratory, DNA Research Centre, Poznań, Poland; Laboratory of High Throughput Technologies, Institute of \\ Molecular Biology and Biotechnology, Faculty of Biology, Adam Mickiewicz University, Poznan, Poland \\ $A$ - research concept and design; $B$ - collection and/or assembly of data; $C$ - data analysis and interpretation; \\ $D$ - writing the article; $E$ - critical revision of the article; $F$ - final approval of article.
}

Gózd-Barszczewska A, Kozioł-Montewka M, Barszczewski P, Młodzińska A, Humińska K. Gut microbiome as a biomarker of cardiometabolic disorders. Ann Agric Environ Med. 2017; 24(3): 416-422. doi: 10.26444/aaem/75456

\begin{abstract}
Introduction and objective. Cardiovascular diseases are the leading cause of death in Europe and worldwide. One of the most important risk factors for atherosclerosis are lipid metabolism disorders, in particular hipercholesterolaemia. The aim of the study was to determine the correlation between gut microbiota composition and atherosclerosis risk factors, so in order that it might be used as a biomarker for coronary artery disease diagnosis.

Material and method. The study involved middle-aged men in eastern Poland with central obesity ( $n=20)$, subjects with atherosclerosis $(n=15)$ and those with no cardiovascular diseases $(n=5)$. The gut microbiota composition was determined using tag-encoded 16S rRNA gene using Illuminal MiSeq. Data were analyzed with the use of t-test.

Results. Firmicutes (49.26\%) and Bacteroidetes (44.46\%) were the dominant Phyla in the middle-aged men in eastern Poland. Subjects with improper levels of total cholesterol were enriched in Prevotella $(p=0.03)$ and decreased level of Clostridium $(p=0.02)$. They also showed a falling tendency in Faecalibacterium $(p=0.07)$. An upward trend was observed in Prevotella $(p=0.07)$ in subjects with improper LDL-C values.

Conclusions. The study showed that intestinal microbiome is likely to play a role in the pathogenesis of atherosclerosis through its role in lipid metabolism. Bacterial genera of particular importance were Prevotella, Bacteroides, Clostridium, Faecalibacterium. However, further studies involving larger groups of subjects are required to confirm these observations.
\end{abstract}

\section{Key words}

gut microbiota, atherosclerosis, lipid disorders, microbiome, cardiovascular diseases

\section{INTRODUCTION}

The Lublin province is a low-industrial region in eastern Poland where more than $50 \%$ of the population lives in the countryside [1]. Cardiovascular diseases remain the dominant cause of death in the region, with mortality rates still higher for the rural population. According to data from 2013, in Lublin province, ischemic heart disease was the second most common cardiovascular disease after hypertension [2]. Improper metabolic processes, which play contributory role in the development of certain cardiovascular disorders, are of a complex nature, and atherosclerosis results from the coexistence of a number of different risk factors. Lipid metabolism disorders, especially hypercholesterolaemia, are of critical importance in the development of atherosclerosis and cardiovascular disease (CVD). The first study to provide considerable evidence of a link between elevated lipid levels and coronary artery disease (CAD) was the Framingham Heart Study [3]. The International INTERHEART study

Address for correspondence: Anna Gózd-Barszczewska, Division of Cardiology with the Cardiac Intensive Care Unit, The Cardinal Stefan Wyszyński Hospital in Lublin, Poland, Al. Kraśnicka 100, 20-718 Lublin, Poland

e-mail: aniagozd@o2.pl

Received: 01.06.2017; accepted: 08.06.2017; first published: September.2017 resulted in selecting 9 independent risk factors for CAD, namely abnormal lipids, arterial hypertension, central obesity, diabetes, smoking, psychosocial factors, fruit and vegetable deficiency, alcohol abuse and low physical activity [4]. Most of them are classified as so-called modifiable risk factors among which dyslipidaemia occurs most often. However, all the data proved to be insufficient and CVD pathogenesis has yet to be thoroughly studied. Thus, researchers worldwide focus on finding new mechanisms that would enable more effective diagnostic and treatment methods.

In cardiology, the role of microorganisms has been well documented among patients undergoing surgical procedures, such as pacemaker implantation, artificial heart valves replacement or self-expanding occluders closing defects in the heart [5]. The development of molecular biology methods, such as next-generation sequencing (NGS), has resulted in the improvement of knowledge on human microbiome diversity. The use of NGS in clinical practice may allow for more accurate, faster and cost-effective identification of microorganisms [6]. Examining the gut microbiome marks a new approach aimed at finding new agents affecting the onset of lipid metabolism disorders. The microbiome is referred to as a "virtual endocrine organ"[7], due to the role it plays in the synthesis of short-chain fatty acids (SCFA), amino acids, 
vitamins and metabolites, affecting also the processes taking place outside the digestive tract. The interaction between the digestive tract microbes and the host is one of the factors which, to a great extent, determine the patient's condition $[8,9]$. The analyses carried out so far show that changes in gut microbiota composition account for many metabolic disorders, including obesity [10-12], inflammatory bowel diseases $[13,14]$, rheumatoid arthritis $[15,16]$ or type 2 diabetes $[17,18]$. The World Microbiome Project provided information on how race, place of residence, and diet affect gut microbiota composition [19]. Studies carried out in recent years contributed to the discovery of the correlation between changes in the gut microbiota composition and CVD. Microbes could play important role in bile acid conversion and in food amines transformation into a proatherogenic component called trimethylamine-N-oxide (TMAO) [20-22].

The next stage of the research carried out globally focused on determining tight associations between the microbiota composition of a given population and metabolic disorders. Much effort is also being made to assess how possible it is to modify microbiome composition so as to promote beneficial microbiome members with a view to developing prevention and therapeutic strategies [8].

\section{OBJECTIVES}

The eastern region of Poland marks the border of European Union and is the region where different multicultural traditions concerning dietary habits and lifestyles meet. The authors of this study are the first to conduct research in the region on the gut microbiota composition in a group of obese middle-aged men living in this low-industrial region, with the aim of finding a correlation between the gut microbiome and atherosclerosis risk factors. The results were compared with those obtained in other regions of the world in order to identify and determine stable and universal correlations between the microbiota composition and its contributory role in developing CVD. The study showed that the composition of the gut microbiota may differ, depending on lipid parameters. Subjects with improper levels of total cholesterol were enriched in genus Prevotella whereas participants with proper levels of total cholesterol were enriched in Clostridium.

\section{MATERIALS AND METHOD}

The study was conducted between 16.02.2015 - 05.05.2015 in the Division of Cardiology with the Cardiac Intensive Care Unit of the Cardinal Stefan Wyszyński Hospital in Lublin. The study was approved by the Bioethics Committee registered at the Lublin Medical Association, and all procedures performed in the study were in accordance with the Helsinki Declaration and its later amendments. The study group comprised 20 participants, all of whom received written information about the study and were additionally orally informed about its methods and aims. All subjects gave written consent to participate in the study and granted access to their medical records. Before the investigation, each subject completed a form concerning present and past diseases, medication, including antibiotics and probiotics, dietary habits, allergies, physical activity and taking stimulants. Taking antibiotics or probiotics 2 months prior to the study excluded the patients from the test. Each participant had their weight, height, waist and hips measured according to the same anthropometric criteria. The same rules were also applied to the methods of collecting samples.

The study involved middle-aged (45-65) men living in Lublin region. The study group involved subjects with CAD $(n=15)$ and those with no cardiovascular problems $(n=5)$. There were 8 participants who lived in the countryside and 12 subjects who lived in the city. All the participants suffered from central obesity which is considered to give rise to many cardiovascular diseases, metabolic disorders (including lipid metabolism) or diabetes. Diagnostic criteria for overweight/ obesity were based on the guidelines indicated by the World Health Organisation (WHO) and International Diabetes Federation (IDF) $[23,24]$. Studies published so far have proved that the gut microbiome of obese subjects differs from that of the healthy ones $[10,11]$. Hence, the presented study group comprised only subjects meeting the abovementioned criteria for overweight and obesity.

In Poland, overweight and obesity is a common problem. According to the NATPOL 2011 research programme, the problem of overweight concerns 6.5 million people, and obesity was recognized in $25 \%$ of men, which is $5 \%$ more than 10 years ago. The widespread occurrence of lipid metabolism disorders has also become a matter of concern in Poland. It has been reported that $61.1 \%$ of Poles aged $18-79$ have hypercholesterolaemia. Improper levels of total cholesterol (TC) and low-density lipoprotein cholesterol (LDL-C) affects mainly the middle-aged population, which results in high risk of atherosclerosis and its morbid consequences in this age group [25]. Due to the total cardiovascular risk, the study subjects were divided into 2 groups - those with target parameters of lipid metabolism and those with disorders within particular cholesterol fractions. Lipid profile was assessed according to European guidelines [26]. In the treatment of dyslipidemia it is absolutely essential to reduce $\mathrm{LDL}-\mathrm{C}$ concentration to target values, i.e. $<1.8 \mathrm{mmol} / \mathrm{L}$ $(70 \mathrm{mg} / \mathrm{dl}),<2.6 \mathrm{mmol} / \mathrm{l}(100 \mathrm{mg} / \mathrm{dl})$ and $<3.0 \mathrm{mmol} / \mathrm{l}$ $(115 \mathrm{mg} / \mathrm{dl})$ in patients with very high, high or low to moderate CV risk, respectively. Analyses was performed for the following parameters: TC, LDL-C and triglycerides (TG).

Sample collection. Faecal samples were collected from participants who had previously received oral information about the methods of collecting samples for microbiological tests. Samples accepted for testing, either walnut size or $5 \mathrm{ml}$ in the case of loose stool, were placed into aseptic containers coded according to the test protocol. Immediately after being collected, the samples were placed in adequately coded Eppendorf tubes and stored at the temperature of $-80^{\circ} \mathrm{C}$ in the hospital laboratory until transported to the Medgenetix Laboratory in Warsaw. The Eppendorf tubes were kept in polypropylen containers (Profilab s.c.), specially designed for deep freezing. Previous studies $[11,27]$ point to several reasons why faeces prove to be the adequate material to analyse microbiome composition, namely, a non-invasive method of collection, contents rich in microbes, and the fact that the gut microbiome represents differences between individuals. During transport, the material was placed in dry ice.

Blood samples were collected by qualified medical staff in the morning hours from subjects who had not yet had 
their breakfast. The samples were analyzed for morphology, transaminases, C-reactive protein (CRP), TC, LDL-C, TG and high density lipoprotein (HDL) cholesterol. Subjects with CAD had their blood taken during hospitalization in the Division of Cardiology with the Cardiac Intensive Care Unit at the Cardinal Stefan Wyszyński Hospital in Lublin, and from those with no prior history of heart diseases were taken in the hospital laboratory. The parameters of lipid metabolism were measured by means of the Cobas Integra Cobas $\mathrm{C}$ System (Roche Diagnostics Ltd., Switzerland). LDL-C was calculated according to the equation of Friedewald et al. [28].

DNA extraction and quantification / Library preparation and sequencing. DNA was extracted from 180-220 mg stool samples with the QIAamp DNA Stool Mini Kit (Qiagen, Hilden, Germany), according to the manufacturer protocol. The concentration and purity of total DNA isolates in the samples were measured spectrophotometrically at wavelengths of A260 and A280, using a NanoDrop machine (Thermo Scientific, DE, USA). Furthermore, the quantity and quality of DNA was evaluated using a Qubit Fluorometer (Invitrogen, Life Technologies, Grand Island, NY, USA) and agarose gel electrophoresis, respectively. The final DNA concentration was standardized before amplification.

The faecal microbiota composition was determined using tag-encoded 16S rRNA gene amplicon MiSeq-based high throughput sequencing (Illumina, San Diego (CA), USA). Primers targeting V3-V4 hipervariable region of $16 \mathrm{~S}$ rRNA contained additional adapters compatible with Nextera Index Kit (Illumina, CA, USA). The primers sequences were as follows: NXt_388_F: 5'- TCG TCG GCA GCG TCA GAT GTG TAT AAG AGA CAG ACW CCT ACG GGW GGC AGC AG -3' and NXt_518_R: 5'-GTC TCG TGG GCT CGG AGA TGT GTA TAA GAG ACA GAT TAC CGC GGC TGC TGG -3'.

PCR reactions containing $12.5 \mu 12 \mathrm{x}$ KAPA HiFi HotStart ReadyMix kit (KAPA BIOSYSTEMS, USA), $5 \mu$ l of each primer $(1 \mu \mathrm{M}), 2.5 \mu \mathrm{l}$ of genomic DNA $(\sim 5 \mathrm{ng} / \mathrm{ul})$, and nucleasefree water to a total volume of $25 \mu \mathrm{l}$ were run on a C1000Touch thermal cycler (BioRad, CA, USA). Cycling conditions applied were: Denaturation at $95^{\circ} \mathrm{C}$ for $3 \mathrm{~min} ; 25$ cycles of $95^{\circ} \mathrm{C}$ for $30 \mathrm{~s}, 55^{\circ} \mathrm{C}$ for $30 \mathrm{~s}$ and $72^{\circ} \mathrm{C}$ for $30 \mathrm{~s}$; followed by final elongation at $72^{\circ} \mathrm{C}$ for $5 \mathrm{~min}$. To incorporate primers with adapters and indexes, PCR reactions contained $25 \mu \mathrm{l} 2 \mathrm{x}$ KAPA HiFi HotStart ReadyMix kit (KAPA BIOSYSTEMS, USA), $5 \mu$ l corresponding P5 and P7 primer (Nextera Index Kit), $5 \mu \mathrm{l}$ PCR product and nuclease-free water for a total volume of $50 \mu \mathrm{l}$. Cycling conditions applied were: $95^{\circ} \mathrm{C}$ for $3 \mathrm{~min} ; 8 \mathrm{cycles}$ of $95^{\circ} \mathrm{C}$ for $30 \mathrm{~s}, 55^{\circ} \mathrm{C}$ for $30 \mathrm{~s}$ and $72^{\circ} \mathrm{C}$ for $30 \mathrm{~s}$; elongation at $72^{\circ} \mathrm{C}$ for $5 \mathrm{~min}$. The amplified fragments with adapters and tags were purified using AMPure XP beads (Beckman Coulter Genomic, CA, USA). Prior to library pooling, clean constructs were quantified using a Qubit fluorometer (Invitrogen, Carlsbad, CA, USA) and mixed in equimolar concentrations to ensure even representation of reads per sample followed 250 bp pair-ended MiSeq (Illumina, CA, USA) sequencing performed according to the instructions of the manufacturer.

Sequencing data / Diversity and statistical analysis. The raw dataset containing pair-ended reads with corresponding quality scores was merged using PEAR software [29], and trimmed with quality lower than 30 and converted to fasta format using FASTX-Toolkit [30]. The header of each read was relabeled with an index number followed by a sample ID. Purging the dataset from chimeric reads and constructing de novo Operational Taxonomic Units (OTU) with a minimum identity of $97 \%$ were conducted using the UPARSE pipeline [31]. Taxonomy was assigned to representative OTU sequences using uclust [32] implemented in the assign_taxonomy script from Quantitative Insights into Microbial Ecology (QIIME) [33] software package (1.8.0) with $90 \%$ of identity. The Greengenes (13.8) $16 \mathrm{~S}$ rRNA gene collection was used as a reference database [34].

A phylogenetic tree was created by performing PyNAST [33] sequence alignment against GreenGenes database. Alpha and beta diversity were calculated with the use of core diversity workflow. The number of sequences was set to $85 \%$ of the sequence number within the most indigent sample (18,000 reads/sample). Differences in alpha diversity were determined using non-parametric t-test with the default number of permutations (999). Unweighted and weighted UniFrac distance matrices were calculated for principal coordinates analysis (PcoA). Diversity analysis was performed by python scripts implemented in QIIME software.

Samples were pooled into 2 bins (proper/improper) for 3 groups of clinical data (TC, LDL-C and TG). Each group was analyzed separately. Statistical differences between proper/ improper means were evaluated by unpaired, two-tailed t-test or permutation t-test (10,000 permutations) by R package [35]. Data were previously checked for normality by Shapiro-Wilk test. Boxplots were performed by $\mathrm{R}$ package.

\section{RESULTS}

The data obtained from metagenomic analysis were divided into 2 groups defining proper and improper concentration of LDL-C, TC and TG according to European guidelines. Bioinformatics analysis was performed which resulted in $1,199,163$ reads. With Greengenes database, it was found that the majority $(99.81 \%)$ of aligned reads were bacterial. Archea accounted for $0.02 \%$ of reads and $0.17 \%$ reads were marked unassigned. For systematic categories Kingdom, Phylum and Class, we were able to align $100 \%$ reads to suitable bacterial taxa. With Order and Family categories, the alignment reached the value of $99.99 \%(99.3-100 \%)$ and 95.85\% (89.09-99.93\%), respectively, whereas Genus had the total of $75.68 \%$ (50.87-94.76\%). Phylum, Family and Genus were analyzed in terms of differences in microbiome composition. Within Phylum level the reads enabled the identification of 12 microbial phyla (Fig. 1).

Intestinal microbiota was found to be dominated by Firmicutes, Bacteroidetes, Proteobacteria and Actinobacteria which represent $>99 \%$ of total reads, with the first two constituting $49.26 \%$ and $44.46 \%$ of the reads. Within the Phylum level, no significant differences were observed in cholesterol metabolism.

Within the Family level, the reads enabled identification of 45 microbial families. The Family level was dominated by Bacteroidaceae 25.29\%, Ruminococcaceae 20.95\%, Prevotellaceae $12.58 \%$, Lachnospiraceae $12.57 \%$, Veilonellaceae $11.37 \%$ and Enterobacteriaceae $2.93 \%$ (Fig. 2).

Analysis of the Genus level revealed the presence of 67 genera. Microbial abundance in Genus level is presented in Figure 3. Component analysis revealed changes within 


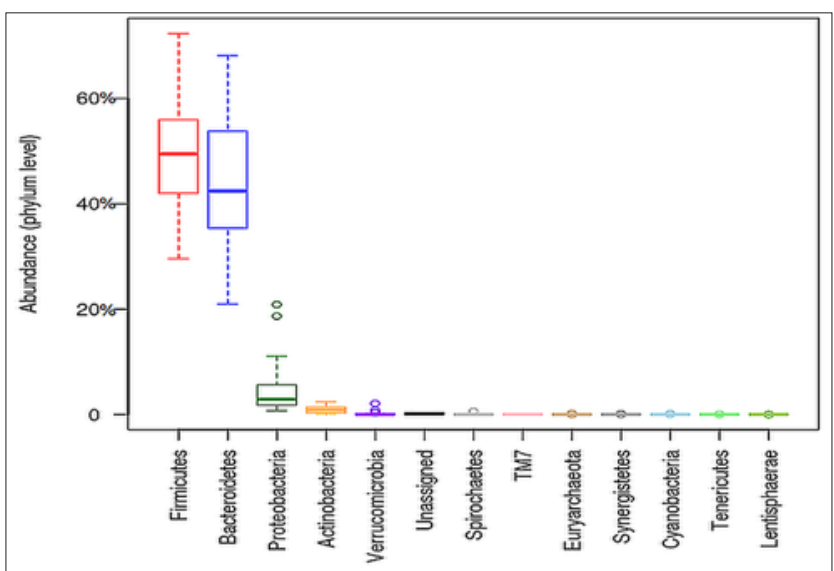

Figure 1. Phylum abundance box plot.

Results indicate the dominance of the phyla Firmicutes and Bacteroidetes. Reads not classified to any taxonomy marked as Unassigned

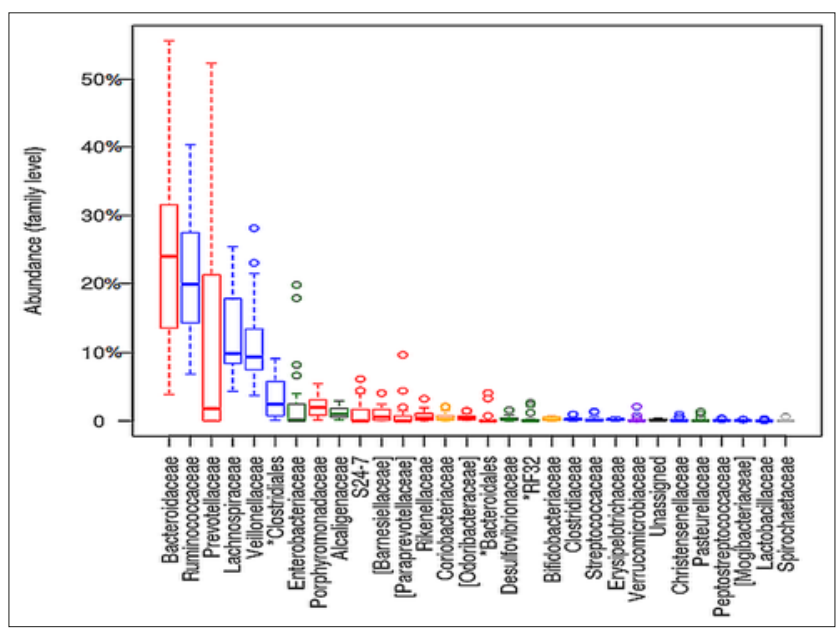

Figure 2. Family abundance variation box plot for the 30 most abundant familie as determined by read abundance.

Families are coloured according to their respective Phylum. Reads not classified to any taxonomy marked Unassigned. Unclassified families under a higher rank marked by asterisks. Results in square brackets represent those suggested by the Greengenes database

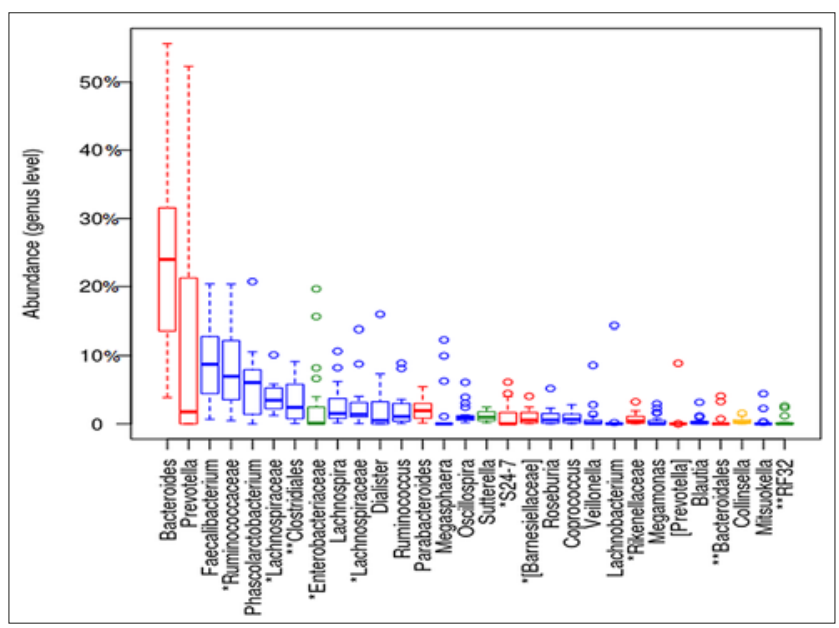

Figure 3. Genus abundance box plot. Genus abundance variation box plot for the 30 most abundant genera as determined by read abundance.

Genera are coloured according to their respective Phylum. Reads classified to any taxonomy marked as unassigned. Unclassified genera under a higher rank marked by asterisks (Family) or double asterisk (Order). Results in square brackets represent those suggested by the Greengenes database microbiota composition according to lipid parameters (Fig. 4). Genus Prevotella was enriched in patients with improper LDL-C level vs. those with proper level (18.20\% vs. $4.15 \%, \mathrm{t}=-1.89 ; \mathrm{p}=0.07)$, whereas Bacteroides levels appeared lower (21.65\% vs. $30.75 \%, t=1.39 ; \mathrm{p}=0.18)$ Fig. 5.

More statistical differences were observed in the analysis of TC concentration. Patients with improper TC levels showed higher values of Prevotella ( $24.61 \%$ vs $7.42 \%, t=-2.24 ; \mathrm{p}=0.03$ ) and lower values of Bacteroides (17.03\% vs $28.83 \%, \mathrm{t}=1.73$; $\mathrm{p}=0.1$ ), compared with patients meeting the criteria for proper TC concentration (Fig. 6). Participants characterized with proper TC levels demonstrated a significantly increased level of genus Clostridium ( $\mathrm{t}=2.47 ; \mathrm{p}=0.02$ ) (Fig. 7). It was also observed that genus Faecalibacterium showed a falling tendency in patients with improper TC level. In proper TC level subjects, genus Faecalibacterium represented 10.80\% of aligned reads, whereas in improper TC level patients it accounted for only $5.84 \%(\mathrm{t}=1.91 ; \mathrm{p}=0.07)$ of reads (Fig. 8)

TG analysis did not reveal any statistically significant differences between genus Prevotella and Bacteroides in both proper and improper TG levels (Prevotella $\mathrm{t}=0.12$; $\mathrm{p}=0.89$, Bacteroides $(\mathrm{t}=-0.64 ; \mathrm{p}=0.52)$. Genus Dialister was detected more often in participants with improper levels of TG $(t=-1.78 ; \mathrm{p}=0.07)$. In the current study, as in previously published reports [36], a higher Bacteroides level was accompanied by an increased level of Parabacteroides, while increase in Prevotella was accompanied by a higher level of Desulfovibrio.

\section{DISCUSSION}

The presented study indicates that a higher abundance of Prevotella co-occurs with TC and LDL-C level disorders, and these are cholesterol fractions most responsible for CVD $[37,38]$. On the basis of studies published in recent years, carried out both on human and animal models, it can be concluded that microbes can contribute to the development of atherosclerosis via food amines metabolism and the process of bile acids transformation. The amines, which include choline, phosphatidylcholine and L-carnitine, are converted to trimethylamine (TMA) which, through oxidation processes with hepatic flavin monooxygenases, is further converted to trimethylamine-N-oxide (TMAO) - regarded as a proatherogenic agent $[21,39,40]$. TMAO is reported to inhibit reverse cholesterol transport (RCT) and stimulate cholesterol accumulation in macrophages. Activated macrophages including LDL, referred to as foam cells, become decomposed due to accumulating excess cholesterol, which consequently leads to atherosclerosis plaque formation. Tang et al. reported that during 3 years observation, patients with elevated TMAO appeared to be more exposed to long-term risk of major adverse cardiovascular events [41]. The authors pointed out that elevated L-carnitine level can be associated with CVD only when accompanied by higher TMAO level. Not all microbes show the same ability to synthesize TMAO, which may be a decisive factor in the assessment of microbiome contribution in CVD pathogenesis. To-date, it has been stated that Prevotella are more efficient at producing TMAO than Bacteroides [21].

The current study indicates that patients with improper TC levels tend to have significantly higher Prevotella values than those with proper TC levels $(\mathrm{p}=0.03)$. The tendency 


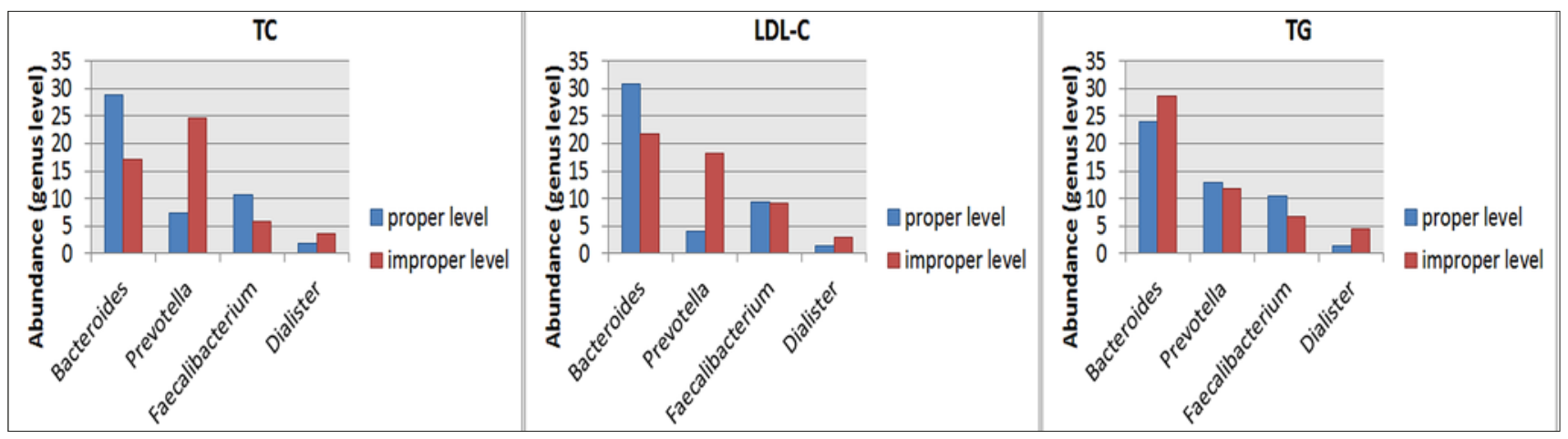

Figure 4. Alterations in Genus abundance depending on the parameters of lipid metabolism. TC - total cholesterol; LDL-C - low-density lipoprotein cholesterol; TG - triglycerides. Proper level - subjects meeting the criteria for proper lipid metabolism; Improper level - subjects not meeting criteria for proper lipid metabolism
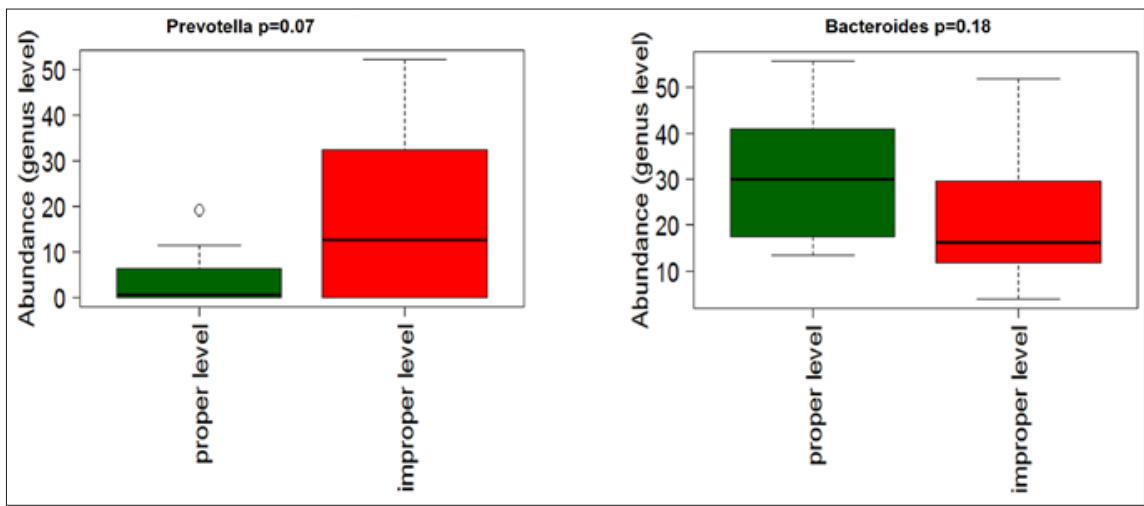

Figure 5. Proper and improper levels of subjects meeting and not meeting the criteria for proper lipid metabolism in LDL-C. The results show a tendency towards increased Prevotella values in subjects with improper LDL-C results $(t=-1.89 ; p=0.07)$
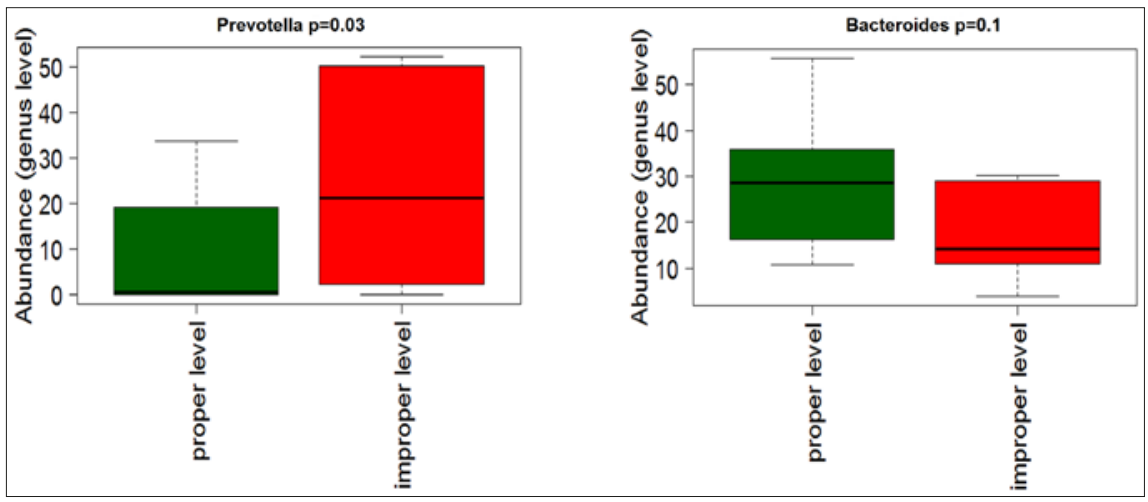

Figure 6. Proper and improper levels of subjects meeting and not meeting the criteria for proper lipid metabolism in TC. The results show significantly higher values of Prevotella in subjects with improper TC values $(t=-2.24 ; p=0.03)$

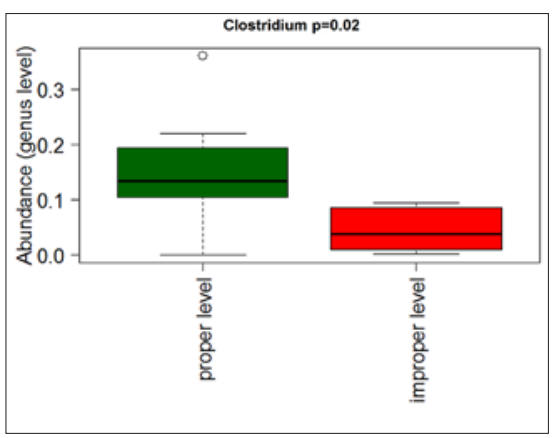

Figure 7. Genus Clostridium abundance. Proper level - subjects meeting the criteria for proper lipid metabolism in TC. Improper level - subjects not meeting criteria for proper lipid metabolism in TC. Results show a significantly increased abundance in Clostridium values in subjects with proper TC results $(\mathrm{t}=2.47 ; \mathrm{p}=0.02)$

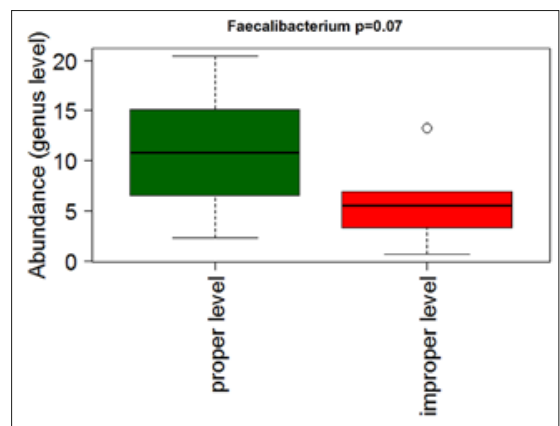

Figure 8. Genus Faecalibacterium abundance. Proper level - subjects meeting the criteria for proper lipid metabolism in TC. Improper level - subjects not meeting criteria for proper lipid metabolism in TC. Results show tendency towards decreasing Faecalibacterium values in subjects with improper TC results $(t=1.91 ; p=0.07)$ 
to higher abundance of Prevotella was also observed in patients with improper levels of LDL-C fraction $(\mathrm{p}=0.07)$. Analysis of the findings for the current study, also showed a significantly higher abundance of genus Clostridium in subjects with proper TC values $(\mathrm{p}=0.02)$. Karlsson et al. [20] demonstrated that genus Clostridium correlate negatively with hs-CRP values, which is a generally recognized marker of an inflammatory condition in CVD. From the clinical point of view, LDL-C and TC are cholesterol fractions which are most highly related to the development of atherosclerosis. Many studies point to the fact that decrease in LDL-C levels reduces the risk of $\mathrm{CVD}$, which is absolutely essential in terms of its prevention [42]. In the presented study, genus Faecalibacterium showed a falling tendency in participants with improper TC levels $(\mathrm{p}=0.07)$. Genus Faecalibacterium is represented by Faecalibacterium prausnitzii which is one of the most abundant butyrate-producing bacterium in the gastrointestinal tract. Butyric acid is an important source of energy for intestinal epithelium cells and the intestinal effects of its activities include the improvement of inflammation, oxidative status, epithelial defence barrier regulation, and the modulation of visceral sensitivity and intestinal motility [43]. Moreover, the global effect of butyrate is to downregulate the expression of 9 key genes involved in intestinal cholesterol biosynthesis, potentially inhibiting this pathway [44].

The results obtained in the current study confirm those reported by Dethlefsen et al. [45] who presented microbiome as a community of microbes dominated by the representatives of several dominant taxa, and accompanied by a number of less abundant taxa ("long tail effect") (Figs. 1-3). The sequencing methods led to a fairly detailed recognition of intestinal microbiome composition at Phylum level. The current results indicate that gut microbiota is considerably dominated by phyla Firmicutes and Bacteroidetes, which is in agreement with previously published data $[20,36,46,47]$. The authors share the opinion presented by Arumugam et al. [36] that there is a growing worldwide consensus concerning gut microbiome composition at Phylum level. Qin et al. proved that $40 \%$ of microbe genes of an individual can also be found in half of the overall population, and thus the term "core microbiome" was coined [48]. Previous and current research by the authors of the presented study confirm that in order to discover the role of microbiota in sustaining systemic homeostasis, analyse of the microbiome at a lower level of taxonomic categories should be undertaken. The way microbes affect our condition does not result only from their quantity; it is also important how they relate to one another and how the products of their metabolism affect our condition.

It should be emphasized that the role of microbiota in pathological processes results from the products of microbe metabolism. The authors hope that the observations they have made so far may, to some extent, contribute to improving the diagnostic methods for atherosclerosis. This particularly concerns people with CVD risk factors but with no clinical manifestations of the disease. In patients with CAD treated with hypolipemizing therapy with statins, which did not achieve the desired therapeutic results, it is worthwhile considering the proatherogenic composition of gut microbiota as a possible cause of the problem. Contemporary modern methods of scientific investigation, including DNA sequencing, gave rise to incredible progress in microbiology and provided access to knowledge previously unattainable. The problems faced now by researchers involve determining the correlation between molecular study results and the clinical condition of the patients. To-date, it has been suggested that gut microbiota plays a significant role in maintaining systemic homeostasis due to its function in nutrition, metabolism and immunological processes [49]. The authors believe that there is a need to conduct further studies involving larger groups to confirm the presented observations. Hence, it is advisable to follow the suggestions by Vinje et al. and apply Koch's postulates to decide whether the gut microbiota compositional modifications result from or in cardiometabolic disorders [50].

\section{CONCLUSIONS}

The results obtained in this study indicate that intestinal microbiome is likely to play a role in the pathogenesis of atherosclerosis through the role it plays in lipid metabolism. Special attention is paid to the bacterial genera Prevotella, Bacteroides, Clostridium, Faecalibacterium which present characteristic changes in patients with lipid disorders. Hence, there is a need for further studies to establish how pharmacological and dietary intervention affects gut microbiota, to determine whether there is a possibility to regulate the microbiome composition in CVD therapy.

\section{Acknowledgments}

The authors wish to express their thanks Ryszard Grzywna, head of the Division of Cardiology with the Cardiac Intensive Care Unit, at the Cardinal Stefan Wyszyński Hospital in Lublin where the study was conducted, and to the DNA Research Centre for the "wet lab" work - especially Marta Kuś-Słowińska (laboratory diagnostician), Magdalena Garbowicz and Jacek Wojciechowicz (CEO).

\section{Ethical approval}

All procedures performed in this study were in accordance with the ethical standards of the Institutional Research Committee and with the 1964 Helsinki Declaration and its later amendments, or comparable ethical standards.

\section{REFERENCES}

1. Urząd Statystyczny w Lublinie: Ludność - dane wojewódzkie. Retrieved from: http://lublin.stat.gov.pl/dane-o-wojewodztwie/ wojewodztwo-895/ludnosc-935/. [Accessed 18 April 2017].

2. Lubelski Urząd Wojewódzki w Lublinie: Stan zdrowia ludności II rozdział Informatora Statystycznego Ochrony Zdrowia Województwa Lubelskiego za rok 2013. Retrieved from: www.lublin.uw.gov.pl/ dzialalnosc-kontr/stan-zdrowotny-ludności-ii-rozdział-informatorastatystycznego-ochrony-zdrowia. [Accessed 18 April 2017].

3. Kennel WB, Dawber TR, Kagan A, Rvotskie N, Stokes J $3^{\text {rd }}$. Factors of risk in the development of coronary heart disease--six year follow-up experience. The Framingham Study. Ann Intern Med. 1961; 55: 33-50.

4. Yusuf S, Hawken S, Ounpuu S, Dans T, Avezum A, Lanas F, et al. Effect of potentially modifiable risk factors associated with myocardial infarction in 52 countries (the INTERHEART study): case-control study. Lancet. 2004; 364 (9438): 937-952.

5. Kozioł-Montewka M, Pańczuk A, Tokarska-Rodak M, et al. Current infectious threats associated with the development of civilization and progress in medicine - methods of prevention and education. Health Problems of Civilization. 2015; 1 (8): 6-14.

6. Weiner MP, Kubajka M. The importance of metagenomics research in human ecological niches and their role in the diagnosis of noninfectious diseases. Health Problems of Civilization. 2015; 2 (9): 43-49. 
7. Bocci V. The neglected organ: bacterial flora has a crucial immunostimulatory role. Perspect Biol Med. 1992; 35(2): 251-260.

8. Fujimura KE, Slusher NA, Cabana MD, Lynch SV. Role of gut microbiota in defining human health. Expert Rev Anti Infect Ther. 2010; 8(4): 435-454. doi: 10.1586/ero.10.14.

9. Sekirov I, Russell SL, Antunes LC, Finlany BB. Gut microbiota in health and disease. Physiol Rev. 2010; 90(3): 859-904. doi: 10.11.52/ physrev.00045.2009.

10. Ley RE, Turnbaugh PJ, Klein S, Gordon JI. Microbial ecology: Human gut microbes associated with obesity. Nature. 2006; 444(7122): 1022-3.

11. Turnbaugh PJ, Hamady M, Yatsunenko T, et al. A core gut microbiome in obese and lean twins. Nature. 2009; 457(7228): 480-484.

12. Turnbaugh PJ, Ley RE, Mahowald MA, Magrini V, Mardis ER, Gordon JI. An obesity-associated gut microbiome with increased capacity for energy harvest. Nature 2006; 444(7122): 1027-1031.

13. Greenblum S, Turnbaugh PJ, Borenstein E. Metagenomic systems biology of the human gut microbiome reveals topological shifts associated with obesity and inflammatory bowel disease. Proc Natl Acad Sci USA. 2012; 109(2): 594-599. doi: 10.1073/pnas.1116053109.

14. Frank DN, St. Amand AL, Feldman RA, Boedeker EC, Harpaz N, Pace NR. Molecular-phylogenetic characterization of microbial community imbalances in human inflammatory bowel diseases. Proc Natl Acad Sci. USA. 2007; 104(34): 13780-13785. doi: 10.1073/pnas.0706625104.

15. Scher JU, Abramson SB. The microbiome and rheumatoid arthritis. Na Rev Rheumatol. 2011; 7(10): 569-578. doi: 10.1038/nrrheum.2011.121.

16. Wu X, He B, Liu J, Feng H, Ma Y, Li D, et al. Molecular Insights into Gut Microbiota in Rheumatoid Arthritis. Int J Mol Sci. 2016; 17(3): 431. doi: 10.3390/ijms17030431.

17. Qin J, Li Y, Cai Z, Li S, Zhu J, Zhang F, et al. A metagenome-wide association study of gut microbiota in type 2 diabetes. Nature 2012; 490(7418): 55-60. doi: 10.1038/nature11450.

18. Karlsson FH, Tremaroli V, Nookaew I, Bergström G, Behre CJ, Fagerberg B, et al. Gut metagenome in European women with normal, impaired and diabetic glucose control. Nature 2013; 498(7452): 99-103. doi: 10.1038/nature12198.

19. Human Microbiome Project Consortium. Structure, function and diversity of the healthy human microbiome. Nature 2012; 486(7402): 207-214. doi: 10.1038/nature11234.

20. Karlsson FH, Fåk F, Nookaew I, Tremaroli V, Fagerberg B, Petranovic $\mathrm{D}$, et al. Symptomatic atherosclerosis is associated with an altered gut metagenome. Nat Commun. 2012; 3: 1245. doi: 10.1038/ncomms2266.

21. Koeth RA, Wang Z, Levison BS, Buffa JA, Org E, Sheehy BT, et al. Intestinal microbiota metabolism of L-carnitine, a nutrient in red meat, promotes atherosclerosis. Nat Med. 2013; 19: 576-585. doi.10.1038/ nm.3145.

22. Wang Z, Klipfell E, Bennett B, Koeth R, Levison BS, DuGar B, et al. Gut flora metabolism of phosphatidylcholine promotes cardiovascular disease. Nature 2011; 472(7341): 57-63. doi: 10.1038/nature09922.

23. World Health Organization: Physical status: the use and interpretation of anthropometry. Report of a WHO Expert Committee. World Health Organ Tech Rep Ser. 1995; 854: 1-452. Retrieved from: http://apps.who. int/iris/bitstream/10665/37003/1/WHO_TRS_854.pdf. [Accessed 25 September 2016].

24. Alberti G, Zimmet P, Shaw J. Metabolic syndrome - a new world - wide definition. A Consensus Statement from the International Diabetes Federation. Diabet Med. 2006; 23: 469-480.

25. Zdrojewski T, Solnica B, Cybulska B, Bandosz P, Rutkowski M, Stokwiszewski J, et al. Prevalence of lipid abnormalities in Poland. The NATPOL 2011 survey. Kardiol Pol. 2016; 74: 213-223.

26. Catapano AL, Graham I, De Backer G, Wiklund O, Chapman MJ, Drexel $\mathrm{H}$, et al. ESC/EAS Guidelines for the management of dyslipidaemias: The Task Force for the management of dyslipidaemias of the European Society of Cardiology (ESC) and the European Atherosclerosis Society (EAS) developed with the special contribution of the European Assocciation for Cardiovascular Prevention \& Rehabilitation (EACPR). Eur Heart J. 2016; 253: 281-344.

27. Eckburg BP, Bik EM, Bernstein CN, Purdom E, Dethlefsen L, Sargent $\mathrm{M}$, et al. Diversity of the human intestinal microbial flora. Science 2005; 308(5728): 1635-1638.

28. Friedewald WT, Levy RI, Fredrickson DS. Estimation of the concentration of low-density lipoprotein cholesterol in plasma, without use of the preparative ultracentrifuge. Clin Chem. 1972;18(6): 499-502.

29. Zhang J, Kobert K, Flouri T, Stamatakis A. PEAR: A fast and accurate Illumina Paired-End reAd mergeR. Bioinformatics 2014; 30(5): 614-620, 2014. doi: 10.1093/bioinformatics/btt593.
30. FASTQ/A short-reads pre-processing tools. Retrieved from: http:// hannonlab.cshl.edu/fastx_toolkit/ (version 3.3.2). [Accessed 15 July 2016]

31. Edgar RC. UPARSE: highly accurate OTU sequences from microbial amplicon reads. Nat Methods. 2013; 10(10): 996-998. doi:10.1038/ nmeth.2604.

32. Edgar RC. Search and clustering orders of magnitude faster than BLAST. Bioinformatics 2010; 26(19): 2460-2461. doi: 10.1093/bioinformatics/ btq461.

33. Caporaso JG, Kuczynski J, Stombaugh J, Bittinger K, Bushman FD, Costello EK, et al. QIIME allows analysis of high-throughput community sequencing data. Nat Methods. 2010; 7(5): 335-336. doi: 10.1038/nmeth.f.303.

34. McDonald D, Price MN, Goodrich J, Nawrocki EP, DeSantis TZ, Probst A, et al. An improved Greengenes taxonomy with explicit ranks for ecological and evolutionary analyses of bacteria and archaea. ISME J. 2012; 6(3): 610-618. doi: 10.1038/ismej.2011.139.

35. R Development Core Team. R: A language and environment for statistical computing. R Foundation for Statistical Computing, Vienna, Austria 2008. ISBN 3-900051-07-0, Retieved from: http://www.Rproject.org. (version 0.0.14). [Accessed 17.07.2016].

36. Arumugam M, Raes J, Pelletier E, Le Paslier D, Yamada T, Mende DR, et al. Enterotypes of the human gut microbiome. Nature 2011; 473 (7346): 174-180. doi: 10.1038/nature09944. Epub 2011 Apr 20.

37. Berliner JA, Navab M, Fogelman AM, Frank JS, Demer LL, Edwards PA, et al. Atherosclerosis: basic mechanisms. Oxidation, inflammation, and genetics. Circulation 1995; 91(9): 2488-2496.

38. Zdrojewski T, Jankowski P, Bandosz P, Bartuś S, Chwojnicki K, Drygas $\mathrm{W}$, et al. Nowa wersja systemu oceny ryzyka sercowo-naczyniowego i tablic SCORE dla populacji Polski. Kardiol Pol. 2015; 73: 958-961.

39. Warrier M, Shih D, Burrows A, Ferguson D, Gromovsky AD, Brown $\mathrm{AL}$, et al. The TMAO-Generating Enzyme Flavin Monooxygenase 3 is a Central Regulator of Cholesterol Balance. Cell Rep. 2015; 10(3): 326-338. doi: 10.1016/j.celrep.2014.12.036.

40. Roager HM, Licht TR, Poulsen SK, Larsen TM, Bahl MI. Microbial Enterotypes, Inferred by the Prevotella-to-Bacteroides Ratio, Remained Stable during a 6-Month Randomized Controlled Diet Intervention with the New Nordic Diet. Appl Environ Microbiol. 2014; 80(3): 11421149. doi: 10.1128/AEM.03549-13.

41. Tang WH, Wang Z, Levison BS, Koeth RA, Britt EB, Fu X, et al. Intestinal microbial metabolism of phosphatidylcholine and cardiovascular risk. N Engl J Med. 2013; 368(17): 1575-1584. doi: 10.1056/NEJMoa1109400.

42. Piepoli MF, Hoes AW, Agewall S, Albus C, Brotons C, Catapano AL, et al. European Guidelines on cardiovascular disease prevention in clinical practice: The Sixth Joint Task Force of the European Society of Cardiology and Other Societies on Cardiovascular Disease Prevention in Clinical Practice (constituted by representatives of 10 societies and by invited experts) Developed with the special contribution of the European Association for Cardiovascular Prevention \& Rehabilitation (EACPR). Atherosclerosis 2016; 252: 207-274. doi: 10.1093/eurheartjehw106.

43. Canani RB, Di Costanzo M, Leone L, Pedata M, Meli R, Calignano A. Potential beneficial effects of butyrate in intestinal and extraintestinal diseases. World J Gastroenterol. 2011; 17(12): 1519-1528. doi: 10.3748// wjg.v17.i12.1519.

44. Alvaro A, Solà R, Rosales R, Ribalta J, Anguera A, Masana L, et al. Gene expression analysis of a human enterocyte cell line reveals downregulation of cholesterol biosynthesis in response to short-chain fatty acids. IUBMB Life. 2008; 60(11): 757-764. doi: 10.1002/iub.110.

45. Dethlefsen L, Huse S, Sogin ML, Relman DA. The Pervasive Effects of an Antibiotic on the Human Gut Microbiota, as Revealed by Deep 16S rRNA Sequencing. PLoS Biol. 2008; 6(11): e280. doi: 10.1371/journal. pbio.0060280.

46. Tap J, Mondot S, Levenez F, Pelletier E, Caron C, Furet JP, et al. Towards the human intestinal microbiota phylogenetic core. Environ Microbiol. 2009; 11(10): 2574-2584. doi: 10.1111/j.1462-2920.2009.01982.x.

47. Lozupone CA, Stombaugh JI, Gordon JI, Jansson JK, Knight R. Diversity, stability and resilience of the human gut microbiota. Nature 2012; 489(7415): 220-230. doi: 10.1038/nature11550.

48. Qin J, Li R, Raes J, Arumugam M, Burgdorf KS, Manichanh C, et al. A human gut microbial gene catalogue established by metagenomic sequencing. Nature 2010; 464(7285): 59-65. doi: 10.1038/nature08821.

49. Borody TJ, Paramsothy S, Agrawal G. Fecal microbiota transplantation indications, methods, evidence, and future directions. Curr Gastroenterol Rep. 2013; 15(8): 337. doi: 10.1007/s11894-013-0337-1.

50. Vinje S, Stroes E, Nieuwdorp M, Hazel SL. The gut microbiome as a novel cardio-metabolic Ditarget: the time has come. Eur Heart J. 2014; 35(14): 883-887. doi: 10.1093/eurheartj/eht467. 